\title{
Polymer aggregation correlated transition from Schottky-junction to bulk heterojunction organic solar cells
}

\author{
Bin Yang, Zhengguo Xiao, and Jinsong Huang ${ }^{\text {a) }}$ \\ Department of Mechanical and Materials Engineering, and Nebraska Center for Materials and Nanoscience, \\ University of Nebraska-Lincoln, Lincoln, Nebraska 68588-0656, USA
}

(Received 11 March 2014; accepted 30 March 2014; published online 11 April 2014)

\begin{abstract}
The fullerene-based organic Schottky-junction solar cells have recently attracted intensive research interest because of their unique electrical performance, such as significant photocurrent generation from excitons created in fullerenes and large open-circuit voltage $\left(V_{\mathrm{OC}}\right)$ output induced by high Schottky-barrier height between the anode and the fullerene acceptor. This manuscript reports another remarkably appealing advantage that the fullerene-based Schottky-junction solar cells are more stable than the bulk heterojunction counterparts. The better stability is likely due to mitigative polymer photo-oxidation and/or little morphological change of active film in the aged Schottky-junction devices. The transition from Schottky-junction to bulk heterojunction appears at polymer donor loading ratio of $20-25 \mathrm{wt}$ \% by examining the variation in the $V_{\text {OC }}$ with increased loading ratio of the poly(3-hexylthiophene) donor. Multiple experimental evidences, including the absorbance spectrum measurement, photoluminescence study, active film morphology characterization, and charge mobility measurement, conclusively reveal that the transition from Schottky-junction to bulk heterojunction is correlated to the polymer donor aggregation in the active films. (C) 2014 AIP Publishing LLC. [http://dx.doi.org/10.1063/1.4871306]
\end{abstract}

The Schottky-junction barrier between metal electrode and organic semiconductor plays an important role in various organic electronic devices, such as organic light emitting diodes, ${ }^{1}$ organic photovoltaics $(\mathrm{OPVs}){ }^{2}$ organic bistable memories, ${ }^{3}$ and organic field effect transistors, ${ }^{4}$ which typically results in rectification characteristics. ${ }^{5,6}$ The application of Schottky-junction for highly efficient OPVs, however, receives less research attention. It is widely believed that the built-in potential in organic Schottky-junction devices cannot provide enough driving force to separate the tightly bound Frenkel excitons created in organic semiconductors, ${ }^{7-11}$ and most excitons recombine without dissociating into free electrons and holes and consequently reaching two opposite conductive electrodes. In fact, about half a century ago, the early research on OPVs heavily relied on the formation of Schottky-junction which, however, yielded device power conversion efficiency $(P C E)$ as low as $0.1 \% .^{6,7}$ In order to effectively separate the photo-generated Frenkel excitons in organic semiconductors, Tang in 1986 invented a bilayer heterojunction OPV with two organic materials of energy band offset to generate a strong enough local internal electric field. ${ }^{2}$ According to his design, the copper phthalocyanine was deposited as electron donor layer and perylene tetracarboxylic derivative as electron acceptor layer, boosting the PCE of OPV to $1 \% .^{2}$ Heeger et al. in 1995 designed bulk heterojunction (BHJ) OPVs based on a blend film of poly[2-methoxy-5-(2'-ethyl-hexyloxy)-1,4-phenylene vinylene] and fullerene to generate a large donor/acceptor interface area for efficient exciton dissociation, initiating an era of the OPV field. ${ }^{8}$ Currently, most highly efficient OPVs are based on BHJ structure. In these BHJ OPVs, the Frenkel excitons were separated by the photo-induced electron transfer from low-electron-affinity donor to high-electron-

${ }^{a)}$ Electronic mail: jhuang2@unl.edu. Telephone: +1-402-472-2640. affinity acceptor. The open-circuit voltage $\left(V_{\mathrm{OC}}\right)$ in $\mathrm{BHJ}$ OPVs is governed by the energy level difference between the lowest unoccupied molecular orbital (LUMO) of the acceptor and the highest occupied molecular orbital (HOMO) of the donor $\left(E_{\mathrm{DA}}\right) .{ }^{9}$ Recently, the fullerene-based Schottky-junction OPVs have attracted intensive research interest because of their unique electrical performance, such as significant photocurrent generation from excitons created in fullerenes and large $V_{\mathrm{OC}}$ output induced by high Schottky-barrier height between the anode and the fullerene acceptor. ${ }^{10-12}$ The $V_{\mathrm{OC}}$ in the fullerene based Schottky-junction devices is solely determined by the energy level difference between the LUMO of fullerene and the work function of anode. ${ }^{11}$ As a result, the $V_{\text {OC }}$ is potentially maximized and even possibly approaches the magnitude of optical bandgap of fullerene acceptor with using high work function anode.

In order to show whether the $V_{\mathrm{OC}}$ in fullerene based OPVs with loading a small amount of donor, e.g., 5 wt. \% of donor, is governed by the Schottky-barrier height between the anode and the fullerene acceptor, we extensively investigated the reported $V_{\mathrm{OC}}$ in the literatures as a function of $E_{\mathrm{DA}}-0.3$ in fullerene OPVs based on a number of polymer donors and small molecule donors. ${ }^{10-13}$ It was found that the variation of the $V_{\mathrm{OC}}$ versus $E_{\mathrm{DA}}-0.3$ in the fullerene based OPVs with loading $5 \mathrm{wt} . \%$ donors was apparently different from that in the devices with loading 40-50 wt. \% donors. The reported experimental $V_{\mathrm{OC}}$ almost linearly increased with $E_{\mathrm{DA}}-0.3$ in the $40-50 \mathrm{wt} . \%$ donor based OPVs, which is referred to as a typical BHJ mechanism. However, for the $5 \mathrm{wt} \%$ donor based OPVs, the $V_{\text {OC }}$ was almost unchanged with increasing $E_{\mathrm{DA}}-0.3$ in OPVs with using either molybdenum oxide $(\mathrm{MoOx}) /$ fullerene $\left(\mathrm{C}_{70}\right)$ or poly(3,4-ethylenedioxythiophene): poly(styrenesulfonate) (PEDOT:PSS)/[6,6]-phenyl-C71-butyric acid methyl ester $\left(\mathrm{PC}_{70} \mathrm{BM}\right)$ as Schottky-junction contact (Figure 1(a)). This is because the $V_{\mathrm{OC}}$ is solely determined by 
the Schottky-barrier height at anode/fullerene acceptor interface, which is not changed after adding $5 \mathrm{wt}$. \% of any type of donors. This interesting phenomenon was observed not only in the polymer donors based OPV devices, such as poly(3-hexylthiophene) (P3HT), ${ }^{11}$ poly[4,8-bis-substituted-benzo[1,2-b:4,5-b'] dithiophene-2,6-diyl-alt-4-substituted-thieno[3,4-b]thio-phene2,6- diyl] (PBDTTT-C-T), ${ }^{11}$ and fluorine substituted poly[2,3bis-(3-octyloxyphenyl) quinoxaline-5,8-diyl-alt-thiophene-2, 5-diyl] (FTQ), ${ }^{11}$ but also in the organic small molecules based

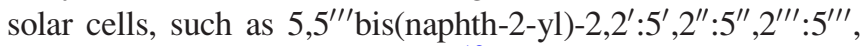
$2^{\prime \prime \prime \prime}$-quinquethiophene (NaT5), ${ }^{12}$ titanyl phthalocyanine (TiOPc) ${ }^{12}$ 1,1-bis-(4-bis(4-methyl-phenyl)-amino-phenyl)-cyclohexane (TAPC), ${ }^{12}$ 5-(2,6-bis((E)-2-(3,4-dioctyl-[2,2 $: 5^{\prime}, 2^{\prime \prime}: 5^{\prime \prime}, 2^{\prime \prime \prime}$ quaterthiophen]-5-yl)vinyl)-4H-pyran-4-ylidene)-1,3-diethyl2-thioxodihydropyrimidine-4,6 (1H, 5H)-dione (8TPDC8), ${ }^{11}$ tetraphenyldibenzoperiflanthene (DBP), ${ }^{13}$ rubrene, ${ }^{12}$ and squaraine. ${ }^{10}$ This discovery also ambiguously indicates that
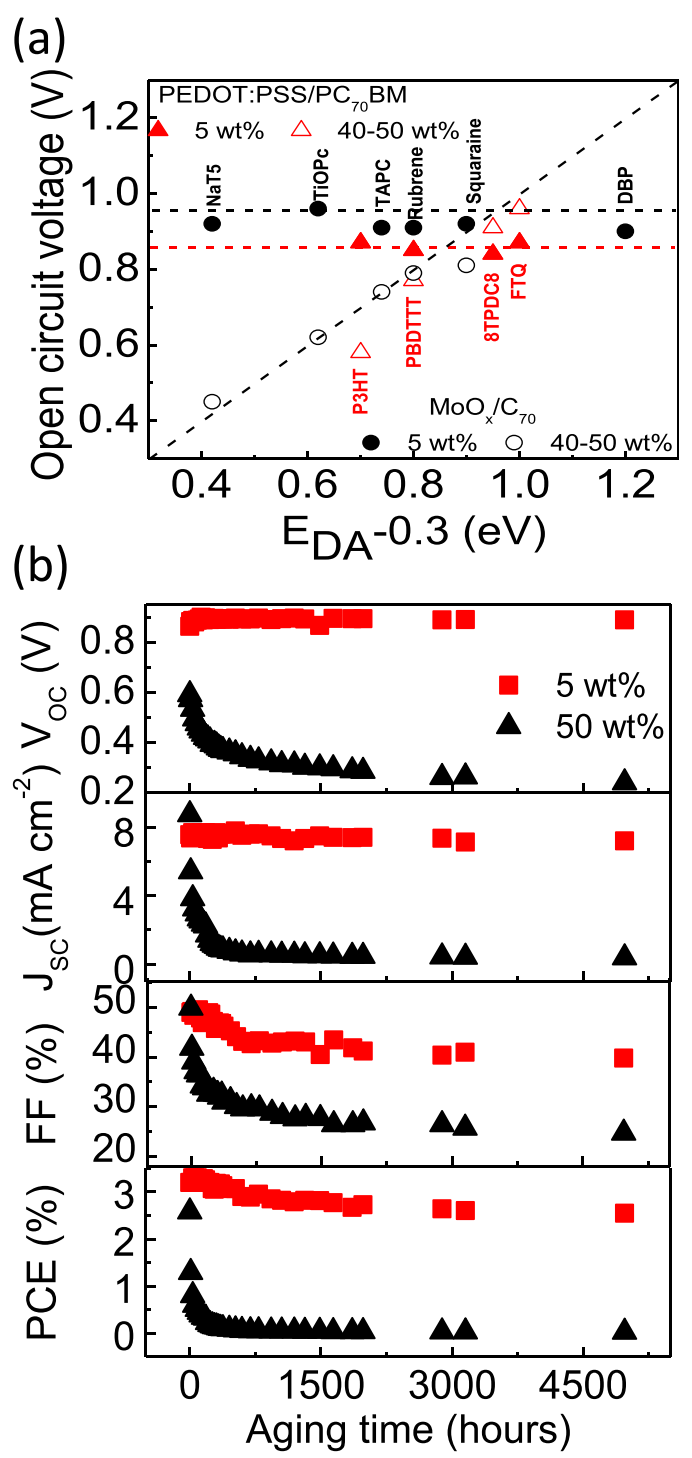

FIG. 1. (a) Variation of the $V_{\mathrm{OC}}$ versus $E_{\mathrm{DA}}-0.3$ in the fullerene based OPVs with loading 5 wt. $\%$ donors and the devices with loading 40-50 wt. \% donors; Red color represents devices with PEDOT:PSS/PC ${ }_{70} \mathrm{BM}$ contact, and black color represents devices with $\mathrm{MoOx} / \mathrm{C}_{70}$ contact. (b) Comparison of device performance between the Schottky-junction solar cells with loading 5 wt. \% P3HT donor and the BHJ devices with loading 50 wt. \% P3HT donors. there should be a transition from Schottky-junction to BHJ with increased donor loading ratio, which needs to be bridged.

Previously, studies found the efficiency of fullerene based Schottky-junction solar cells was comparable to that of the BHJ counterparts. ${ }^{11,12}$ However, the stability of fullerene based Schottky-junction device and physical transition from Schottky-junction to BHJ with increased donor loading ratio were rarely investigated. In this manuscript, we first compared the device stability between the Schottky-junction solar cells and the BHJ devices, and the measured device architectures were indium tin oxide (ITO)/PEDOT:PSS/ 95 wt. \% PC $_{70} \mathrm{BM}: 5$ wt. \% P3HT/bathocuproine (BCP)/ aluminum (Al), and ITO/PEDOT:PSS/50 wt. \% PC ${ }_{70} \mathrm{BM}$ : 50 wt. \% P3HT/BCP/Al, respectively. The studied Schottky-junction device produced an initial $V_{\text {OC }}$ of $0.86 \mathrm{~V}$, a short circuit current $\left(J_{\mathrm{SC}}\right)$ of $7.55 \mathrm{~mA} \mathrm{~cm} \mathrm{~cm}^{-2}$, a fill factor $(F F)$ of $49 \%$, the resulted $P C E$ of $3.2 \%$, which were consistent with the reported values. ${ }^{11}$ For the as-made BHJ counterpart, the output $V_{\mathrm{OC}}$ was $0.59 \mathrm{~V}$, the $J_{\mathrm{SC}}$ was $8.73 \mathrm{~mA} \mathrm{~cm}^{-2}$, the $F F$ was $50 \%$, and the $P C E$ was $2.6 \%$. These two types of devices were stored in the nitrogen glovebox for aging test. As shown in Figure 1(b), the Schottky-junction solar cells were shown to be more stable than the BHJ counterparts. For the BHJ device, all photovoltaic parameters showed a rapid decrease with aging, and a severe burn-in loss followed by a linear decay was clearly observed in the BHJ device, which has been mainly ascribed to high polymer concentration in the active film and the polymer photo-oxidation leading to trap states. ${ }^{14,15}$ Nevertheless, the decay process of the fullerene based Schottky-junction solar cells was uniquely different. Both $V_{\mathrm{OC}}$ and $J_{\mathrm{SC}}$ were almost unchanged after aging for nearly $5000 \mathrm{~h}$, and the only reduced photovoltaic parameter is $F F$, which started showing a gradual decrease after aging for about $200 \mathrm{~h}$. Since the devices were stored in the nitrogen glovebox which has a trace concentration of oxygen at around $1 \mathrm{ppm}$, the degradation in $F F$ was likely caused by degradation of fullerenes, ${ }^{16}$ or by oxidation of metal aluminum electrode, which resulted in high series resistance as suggested by the less steep slopes of current density-voltage $(J-V)$ curves in the aged Schottky-junction devices. As a result, the $P C E$ in Schottky-junction device was remarkably stable until aging for $200 \mathrm{~h}$, and then the $P C E$ almost linearly decreased to $2.5 \%$ after about $5000 \mathrm{~h}$ aging. Previous studies have suggested that aging the $\mathrm{BHJ}$ devices might also cause the morphological change, such as non-ideal phase separation in the active film, ${ }^{17}$ which leads to inefficient exciton separation, ${ }^{18}$ and/or unfavorable charge transport, ${ }^{19}$ and consequently reduced device efficiency. However, only loading a low donor concentration of $5 \mathrm{wt} \%$ into the active films makes the Schottky-junction device more stable, which could be because (1) mitigative polymer photo-oxidation and/or (2) little changed internal morphology in the aged Schottky-junction devices compared to the aged BHJ counterparts.

In order to bridge the physical transition from the Schottky-junction to BHJ OPVs, we systematically studied device performance variation with increased P3HT loading ratio. The device structure is ITO/PEDOT:PSS/PC $70 \mathrm{BM}$ (x wt. \% P3HT)/BCP/Al. Figure 2(a) shows the $J$ - $V$ curves under illumination of simulated air mass 1.5 global irradiation $\left(100 \mathrm{~mW} \cdot \mathrm{cm}^{-2}\right)$, and Figure 2(b) summarizes the variation of 
(a)
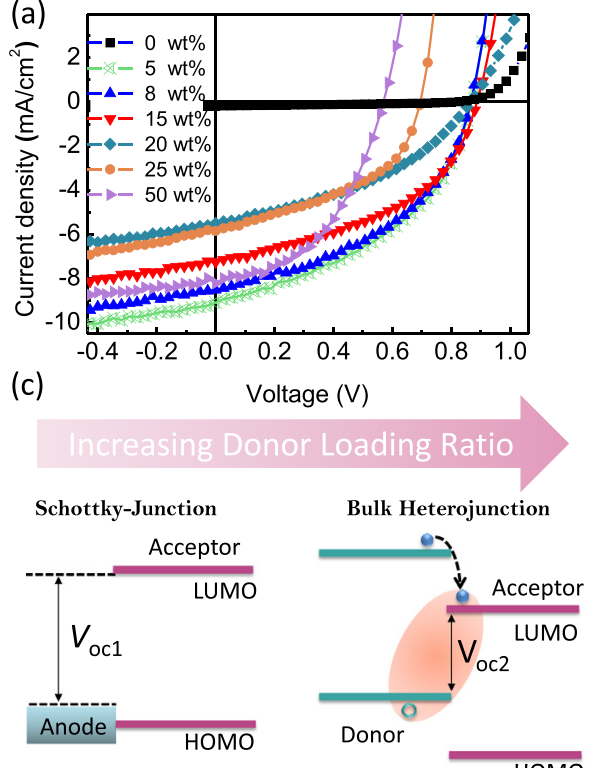

(b)

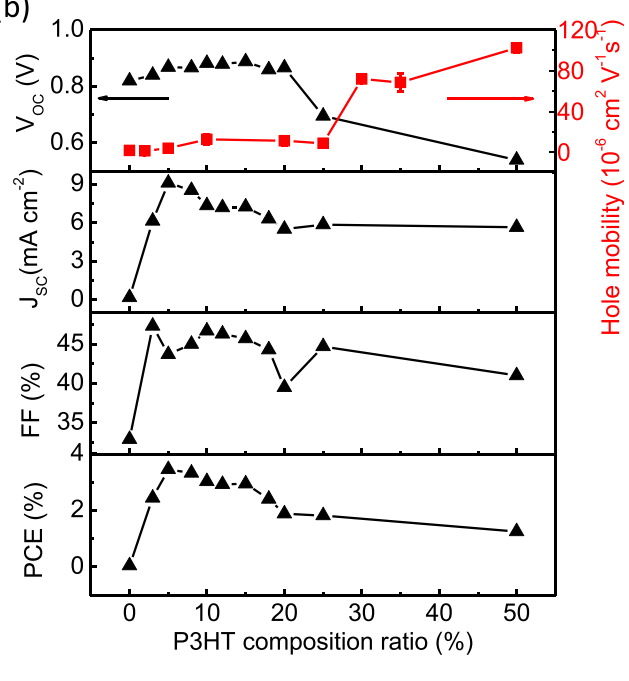

FIG. 2. (a) $J-V$ curves of devices with different P3HT loading ratio under illumination. (b) Variation of photovoltaic parameters and hole mobility with increasing P3HT composition ratio. (c) Schematic diagram of transition from Schottky-junction mechanism to bulk heterojunction mechanism in organic solar cells with increasing donor loading ratio. the obtained photovoltaic parameters with increasing P3HT composition ratio. When the $\mathrm{P} 3 \mathrm{HT}$ composition is smaller than $20 \mathrm{wt} \%$, the $V_{\mathrm{OC}}$ was maintained at $0.85 \pm 0.03 \mathrm{~V}$, which was very close to the one obtained in the pure $\mathrm{PC}_{70} \mathrm{BM}$ based Schottky-junction device. The same $V_{\mathrm{OC}}$ of around $0.85 \mathrm{~V}$ in fullerene based OPVs with loading $0-20$ wt. \% of donor showed that the $V_{\text {OC }}$ is only governed by the Schottky-barrier height between the anode and the fullerene acceptor in these devices, which are suggested as Schottky-junction solar cells (Figure 2(c)). However, with the P3HT composition rising to $25 \mathrm{wt}$ \% , the $V_{\mathrm{OC}}$ dramatically dropped to $0.70 \mathrm{~V}$ and further decreased to $0.54 \mathrm{~V}$ in 50 wt. \% P3HT polymer based OPVs. The decrease in $V_{\text {OC }}$ with increasing P3HT composition from $25 \mathrm{wt}$. \% to $50 \mathrm{wt}$ \% could be thought as an evidence of BHJ mechanism governed devices (Figure 2(c)), because the higher degree of P3HT aggregation or crystallinity at high P3HT composition can lift up the HOMO level of the P3HT and thus reduce the $E_{\mathrm{DA}}{ }^{20}$ The variation in $V_{\mathrm{OC}}$ suggests that the transition from Schottky-junction to BHJ occurs at P3HT composition of approximately $20-25 \mathrm{wt}$. $\%$. The $J_{\mathrm{SC}}$ significantly increased to $9.1 \mathrm{~mA} \mathrm{~cm}^{-2}$ when $5 \mathrm{wt}$. \% P3HT was added into the fullerene materials, which was consistent with our previous results. ${ }^{11}$ Nevertheless, with further increasing the P3HT composition ratio, the $J_{\mathrm{SC}}$ gradually decreased to a saturated value of $6.1 \mathrm{~mA} \mathrm{~cm}^{-2}$. Both the $F F$ and $P C E$ showed a similar variation tendency to that of the $J_{\mathrm{SC}}$. It is worth noting that the OPVs based on P3HT composition of $5 \mathrm{wt}$. \% yielded the highest PCE of about $3.3 \%$ under the illumination of 100 $\mathrm{mW} \cdot \mathrm{cm}^{-2}$, which was consistent with the reported value. ${ }^{11,12}$

The transition from Schottky-junction to BHJ occurs when the P3HT loading ratio was enhanced to 20-25 wt. \%. The next critical step is to reveal the driving force of the transition. In order to do so, we measured the absorption spectra of the active films with different P3HT loading ratio using a UV-Visible Spectrophotometer Evolution 201 (Thermo Scientific). All of the films were spin coated on pre-cleaned quartz glass substrates to minimize measurement error. Figure 3(a) showed the normalized absorption spectra
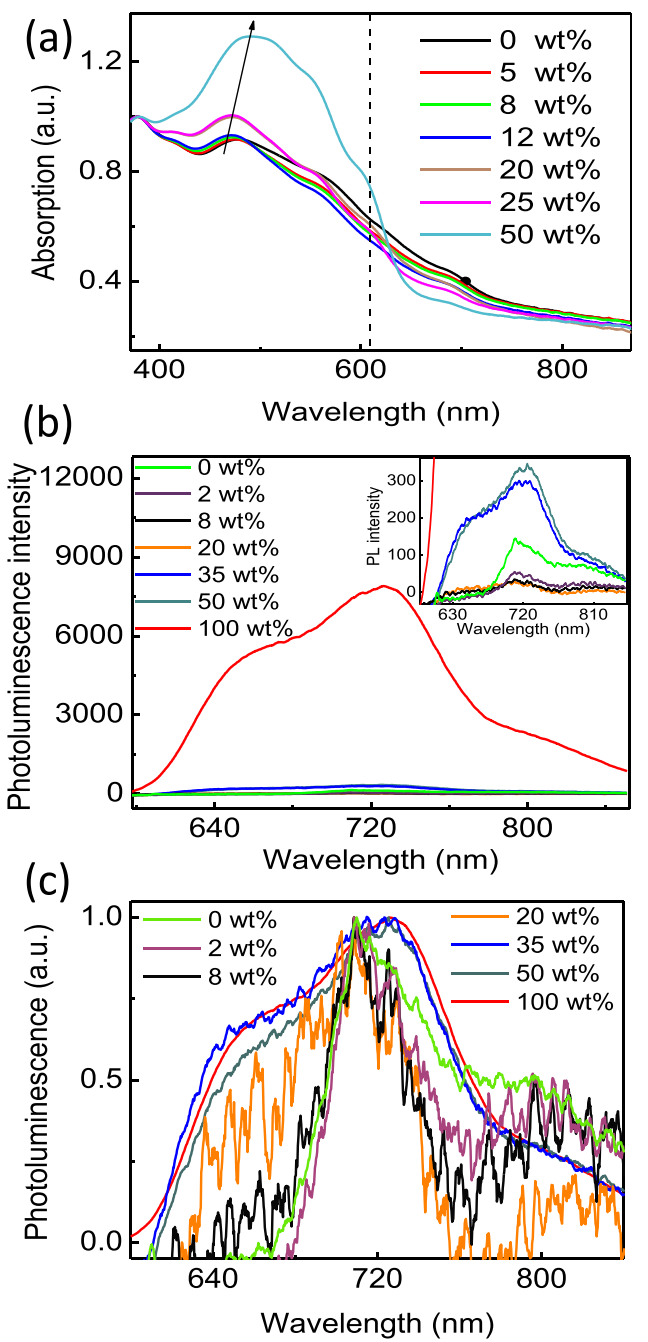

FIG. 3. Variation of absorption spectra (a), photoluminescence spectra (b), and normalized photoluminescence spectra (c) with increased P3HT loading ratio. Inset of (b) is the enlarged photoluminescence spectra. Except 100 wt. \% P3HT sample, all the other photoluminescence spectra were smoothed for better comparison. 
of the $\mathrm{PC}_{70} \mathrm{BM}$ based active films with different P3HT loading ratio. Interestingly, the shape of the absorption spectra of 5 wt. $\%, 8$ wt. $\%, 12$ wt. $\%$, and 20 wt. $\%$ films was found to remain almost same compared to that of the pure $\mathrm{PC}_{70} \mathrm{BM}$ film $(0 \mathrm{wt} . \%)$. This similarity in absorption spectra shape indicates negligible absorption contribution from P3HT polymer. With P3HT composition ratio rising to $25 \mathrm{wt}$ \% , the absorption spectra shape varied, and the characteristic absorption shoulder of P3HT started showing up at around $610 \mathrm{~nm}$. These features signify considerable absorption from the P3HT polymer when the P3HT loading ratio was increased to $25 \mathrm{wt}$ \% $\%$. Furthermore, the $50 \mathrm{wt}$ \% P3HT sample had more pronounced P3HT absorption peaks and shoulders, distinctly indicating higher degree of crystallinity in the P3HT polymer domains. ${ }^{21-23}$ In addition, the absorption peak of the $50 \mathrm{wt}$ \% P3HT film was at about $496 \mathrm{~nm}$, which exhibited an evident red-shift of about $25 \mathrm{~nm}$ compared to that of the $25 \mathrm{wt}$ \% P3HT sample. This red-shift should be a result of the aggregation of P3HT polymers, which consequently leads to the enhanced interchain interaction such as $\pi-\pi$ stacking.

Since the P3HT polymer aggregation benefits the charge transport properties due to the increased $\pi-\pi$ stacking interaction, the variation of charge mobility, e.g., hole mobility, therefore indirectly reflects the polymer aggregation with increased P3HT loading ratio. We measured the hole-only devices with the structure of ITO/MoOx/PC ${ }_{70} \mathrm{BM}$ : $\mathrm{x}$ wt. \% $\mathrm{P} 3 \mathrm{HT} / \mathrm{MoOx} /$ silver $(\mathrm{Ag})$, and the space charge limited current (SCLC) method ${ }^{24}$ was used to calculate hole mobilities for the devices with different P3HT polymer composition. Interestingly, the pristine $\mathrm{PC}_{70} \mathrm{BM}$ film can conduct holes with mobility of $1.84 \pm 0.19 \times 10^{-6} \mathrm{~cm}^{2} \mathrm{~V}^{-1} \mathrm{~s}^{-1}$ (Figure 2(b)). The observed hole transporting property of fullerene material is consistent with other study, ${ }^{25,26}$ which showed that the hole mobility in the fullerene material is about two orders smaller than electron mobility measured by linearly increasing voltage technique and organic field-effect transistors measurement. ${ }^{26}$ In our SCLC measurement, the hole mobility was slowly enhanced with increased P3HT loading ratio until $25 \mathrm{wt}$ \% P3HT polymer was loaded. The hole mobility experienced a rapid increase to $7.18 \pm 0.04 \times 10^{-5}$ $\mathrm{cm}^{2} \mathrm{~V}^{-1} \mathrm{~s}^{-1}$ when 30 wt. \% P3HT was loaded, and then gradually increased to $1.02 \pm 0.05 \times 10^{-4} \mathrm{~cm}^{2} \mathrm{~V}^{-1} \mathrm{~s}^{-1}$ for the $50 \mathrm{wt}$ \% P3HT loading. The sharp increase in hole mobility for films with P3HT concentration of higher than 30 wt. \% indicates a strong P3HT polymer aggregation and therefore significantly increased $\pi-\pi$ stacking interaction.

Photoluminescence (PL) study is another independent technique to confirm the $\mathrm{P} 3 \mathrm{HT}$ polymer aggregation in the active films and find the driving force of the transition from Schottky-junction to BHJ. The PL spectra were measured with HR320 Photoluminescence Spectroscopy. All film samples were prepared by spin-coating on pre-cleaned glass substrates. The samples were excited by single wavelength laser $(532 \mathrm{~nm})$, and the PL emission was collected with a sensitive thermoelectric-device-cooled silicon detector. As shown in Figure 3(b), the PL emission peak of pristine $\mathrm{PC}_{70} \mathrm{BM}$ (0 wt. \% P3HT) was at around $709 \mathrm{~nm}$, which was assigned to the singlet exciton emission of $\mathrm{PC}_{70} \mathrm{BM}^{27}$ The $\mathrm{PL}$ of the pristine $\mathrm{PC}_{70} \mathrm{BM}$ film was significantly quenched by adding a trace amount of P3HT polymer of 2 wt. \%. The PL measurement clearly showed that a small amount of P3HT polymer can effectively dissociate the excitons in $\mathrm{PC}_{70} \mathrm{BM}$ materials. For the $8 \mathrm{wt} . \%$ and $20 \mathrm{wt} . \%$ P3HT samples, the PL emission remained quenched, which however, dramatically recovered in $35 \mathrm{wt} . \%, 50 \mathrm{wt} . \%$, and $100 \mathrm{wt} . \%$ P3HT film. The enhanced PL emission should be ascribed to enhanced amount of P3HT polymers. The PL spectra were further normalized to distinguish the PL emission from P3HT, or PCBM, or both. As shown in Figure 3(c), with reducing P3HT composition from $100 \mathrm{wt}$. \% to $35 \mathrm{wt}$ \%, the shape of PL spectra remained almost unchanged, and this is because the PL emission was mainly from P3HT polymer. However, the PL emission peak showed a blue shift of about $19 \mathrm{~nm}$ when the P3HT loading ratio further decreased to 20 wt. \%. More interestingly, the PL spectrum shape was different from that of the $35 \mathrm{wt} . \%, 50 \mathrm{wt} . \%$, and $100 \mathrm{wt} . \%$ P3HT film samples, which might mean the P3HT polymer aggregation was absent in the $20 \mathrm{wt}$. \% active film. With further reducing P3HT loading ratio to $8 \mathrm{wt} . \%, 2 \mathrm{wt} . \%$, and even 0 wt. $\%$, the shape of PL spectra was similar to that of the $20 \mathrm{wt}$ \% film and the PL emission at around 709-800 nm
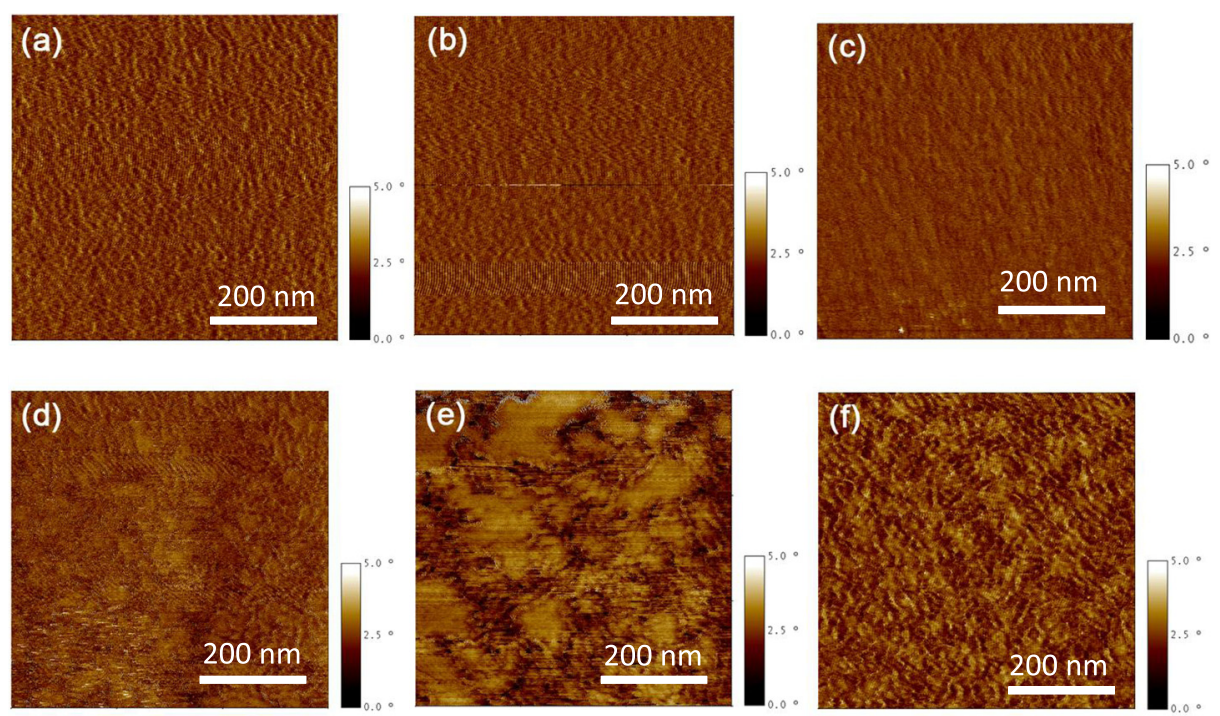

FIG. 4. Variation of active film morphology with increased P3HT loading ratio. The AFM phase contrast images were shown in 0 wt. \% (a), 3 wt. \% (b), 5 wt. $\%$ (c), 12 wt. $\%$ (d), 25 wt. $\%$ (e), and $50 \mathrm{wt} . \%$ (f). 
showed a successive enhancement, which is possibly due to improved PCBM crystallinity. ${ }^{27}$

The above absorption spectra and photoluminescence spectra study apparently indicates that the P3HT polymer starts aggregation at certain P3HT composition of $20-25 \mathrm{wt}$. \%. In order to directly observe the aggregation of the P3HT polymers in the active films, the morphology of the P3HT:PCBM active films was characterized by the atomic force microscopy (AFM) measurement, by which the P3HT and PCBM materials can be differentiated due to their different elasticity and phase angle change. ${ }^{28}$ AFM images were obtained using Multi-mode Nanoscope IIIA (Veeco Metrology Incorporation) with tapping mode. The silicon tip with force constant of $0.5-9.5 \mathrm{~N} / \mathrm{m}$ was from Nanosensors Incorporation (PPP-FM). As shown in Figures 4(a)-4(d), compared to the PCBM-only film ( 0 wt. \%), the AFM phase images of the 3 wt. $\%, 5$ wt. \%, and 12 wt. \% P3HT films are almost unchanged, where the P3HT polymer domain is completely invisible. With further increasing P3HT loading ratio, the aggregation of P3HT polymers was observed in the $25 \mathrm{wt}$ \% $\%$ P3HT film as shown in Figure 4(e). Furthermore, the P3HT aggregation domains grow larger in the $50 \mathrm{wt}$ \% P3HT film (Figure 4(f)), which is well supported by a more pronounced vibronic absorption peak in absorption spectra (Figure 3(a)). Both the emergence of $\mathrm{P} 3 \mathrm{HT}$ polymer aggregation and the sharp decrease in $V_{\mathrm{OC}}$ occur at the same P3HT composition of 20-25 wt. \%, suggesting a strong correlation between the $\mathrm{P} 3 \mathrm{HT}$ aggregation and the physical transition from Schottky-junction to BHJ mechanisms.

In conclusion, the Schottky-junction solar cells were found to be more stable than the BHJ counterparts, which is an attractive merit for potential commercial applications. The transition from Schottky-junction to BHJ occurs at P3HT polymer composition of approximately $20-25 \mathrm{wt}$. \%, providing a common guide to effectively design fullerene based Schottky-junction photovoltaic devices. Multiple evidences, including absorption spectra, hole mobilities, photoluminescence study, and active film morphology, conclusively demonstrated that the transition is strongly correlated to the P3HT polymer aggregation. The underlying detailed physical origin for transition from Schottky-junction to BHJ with increased $\mathrm{P} 3 \mathrm{HT}$ polymer composition is still under investigation.

We thank the financial support by the National Science Foundation under Award Nos. ECCS-1201384 and ECCS1252623, and the Nebraska Public Power District through the Nebraska Center for Energy Sciences Research. We acknowledge Dr. Baodong Mao and Dr. Yongbo Yuan's comments on our manuscript.

${ }^{1}$ P. E. Burrows, Z. Shen, V. Bulovic, D. M. McCarty, S. R. Forrest, J. A. Cronin, and M. E. Thompson, J. Appl. Phys. 79(10), 7991 (1996).

${ }^{2}$ C. W. Tang, Appl. Phys. Lett. 48, 183 (1986).

${ }^{3}$ J. Ouyang, C.-W. Chu, C. R. Szmanda, L. Ma, and Y. Yang, Nature Mater. 3(12), 918 (2004).

${ }^{4}$ M. M. Ling and Z. Bao, Chem. Mater. 16(23), 4824 (2004).

${ }^{5}$ I. H. Campbell and D. L. Smith, Appl. Phys. Lett. 74(4), 561 (1999).

${ }^{6}$ A. K. Ghosh, D. L. Morel, T. Feng, R. F. Shaw, and C. A. Rowe, J. Appl. Phys. 45(1), 230 (1974).

${ }^{7}$ D. Kearns and M. Calvin, J. Chem. Phys. 29(4), 950 (1958).

${ }^{8}$ G. Yu, J. Gao, J. C. Hummelen, F. Wudl, and A. J. Heeger, Science 270(5243), 1789 (1995).

${ }^{9}$ C. J. Brabec, A. Cravino, D. Meissner, N. Serdar Sariciftci, T. Fromherz, M. T. Rispens, L. Sanchez, and J. C. Hummelen, Adv. Funct. Mater. 11(5), 374 (2001).

${ }^{10} \mathrm{G}$. Wei, S. Wang, K. Renshaw, M. E. Thompson, and S. R. Forrest, ACS Nano 4(4), 1927 (2010).

${ }^{11}$ B. Yang, F. Guo, Y. Yuan, Z. Xiao, Y. Lu, Q. Dong, and J. Huang, Adv. Mater. 25(4), 572 (2013).

${ }^{12}$ M. Zhang, H. Wang, H. Tian, Y. Geng, and C. Tang, Adv. Mater. 23(42), 4960 (2011).

${ }^{13}$ Y.-q. Zheng, W. J. Potscavage, Jr., T. Komino, M. Hirade, J. Adachi, and C. Adachi, Appl. Phys. Lett. 102(14), 143304 (2013).

${ }^{14}$ C. H. Peters, I. T. Sachs-Quintana, W. R. Mateker, T. Heumueller, J. Rivnay, R. Noriega, Z. M. Beiley, E. T. Hoke, A. Salleo, and M. D. McGehee, Adv. Mater. 24(5), 663 (2012).

${ }^{15}$ C. H. Peters, I. T. Sachs-Quintana, J. P. Kastrop, S. Beaupre, M. Leclerc, and M. D. McGehee, Adv. Energy Mater. 1(4), 491 (2011).

${ }^{16}$ M. O. Reese, A. M. Nardes, B. L. Rupert, R. E. Larsen, D. C. Olson, M. T. Lloyd, S. E. Shaheen, D. S. Ginley, G. Rumbles, and N. Kopidakis, Adv. Funct. Mater. 20(20), 3476 (2010).

${ }^{17}$ D. H. Wang, J. K. Kim, J. H. Seo, O. O. Park, and J. H. Park, Sol. Energy Mater. Sol. Cells 101, 249 (2012).

${ }^{18}$ Z. Liu, Q. Liu, Y. Huang, Y. Ma, S. Yin, X. Zhang, W. Sun, and Y. Chen, Adv. Mater. 20(20), 3924 (2008).

${ }^{19}$ B. Yang, Y. Yuan, and J. Huang, J. Phys. Chem. C 118, 5196 (2014).

${ }^{20}$ W. C. Tsoi, S. J. Spencer, L. Yang, A. M. Ballantyne, P. G. Nicholson, A. Turnbull, A. G. Shard, C. E. Murphy, D. D. C. Bradley, and J. Nelson, Macromolecules 44(8), 2944 (2011).

${ }^{21}$ G. Li, V. Shrotriya, J. Huang, Y. Yao, T. Moriarty, K. Emery, and Y. Yang, Nature Mater. 4(11), 864 (2005).

${ }^{22}$ M. Campoy-Quiles, T. Ferenczi, T. Agostinelli, P. G. Etchegoin, Y. Kim, T. D. Anthopoulos, P. N. Stavrinou, D. D. C. Bradley, and J. Nelson, Nature Mater. 7(2), 158 (2008).

${ }^{23}$ B. Yang, Z. Xiao, Y. Yuan, T. V. Jayaraman, J. E. Shield, R. Skomski, and J. Huang, Polymer 54(2), 490 (2013).

${ }^{24}$ V. D. Mihailetchi, J. Wildeman, and P. W. M. Blom, Phys. Rev. Lett. 94(12), 126602 (2005).

${ }^{25}$ K. Tokunaga, H. Kawabata, and K. Matsushige, Jpn. J. Appl. Phys., Part 1 47(5R), 3638 (2008).

${ }^{26}$ A. Pivrikas, M. Ullah, Th. B. Singh, C. Simbrunner, G. Matt, H. Sitter, and N. S. Sariciftci, Org. Electron. 12(1), 161 (2011).

${ }^{27}$ S. Cook, H. Ohkita, Y. Kim, J. J. Benson-Smith, D. D. C. Bradley, and J. R. Durrant, Chem. Phys. Lett. 445(4), 276 (2007).

${ }^{28}$ D.-Y. Khang, J. A. Rogers, and H. H. Lee, Adv. Funct. Mater. 19(10), 1526 (2009). 